Article

\title{
Effects of Phenolic Acids on the Growth and Production of T-2 and HT-2 Toxins by Fusarium langsethiae and F. sporotrichioides
}

\author{
Elena Ferruz ${ }^{1}$, Vessela Atanasova-Pénichon ${ }^{2}$, Marie-Noëlle Bonnin-Verdal ${ }^{2}$, Gisèle Marchegay ${ }^{2}$, \\ Laëtitia Pinson-Gadais ${ }^{2}$, Christine Ducos ${ }^{2}$, Susana Lorán ${ }^{1}$, Agustín Ariño ${ }^{1, *}$, \\ Christian Barreau ${ }^{2}$ and Florence Richard-Forget ${ }^{2}$ \\ 1 Veterinary Faculty, Instituto Agroalimentario de Aragón (IA2), Universidad de Zaragoza-CITA, \\ c/Miguel Servet 177, 50013 Zaragoza, Spain; eferruz@unizar.es (E.F.); sloran@unizar.es (S.L.) \\ 2 MycSA, INRA, 71 Avenue Edouard Bourlaux, CS 20032, 33882 Villenave d'Ornon cedex, France; \\ vatanaso@bordeaux.inra.fr (V.A.-P.); Marie-Noelle.Verdal@bordeaux.inra.fr (M.-N.B.-V.); \\ Gisele.Marchegay@bordeaux.inra.fr (G.M.); lpinson@bordeaux.inra.fr (L.P.-G.); \\ christine.ducos@bordeaux.inra.fr (C.D.); cbarreau@bordeaux.inra.fr (C.B.); fforget@bordeaux.inra.fr (F.R.-F.) \\ * Correspondence: aarino@unizar.es; Tel.: +34-876-554-142 \\ Academic Editor: Mary Fletcher \\ Received: 17 December 2015; Accepted: 30 March 2016; Published: 4 April 2016
}

\begin{abstract}
The effect of natural phenolic acids was tested on the growth and production of T-2 and HT-2 toxins by Fusarium langsethiae and F. sporotrichioides, on Mycotoxin Synthetic medium. Plates treated with $0.5 \mathrm{mM}$ of each phenolic acid (caffeic, chlorogenic, ferulic and $p$-coumaric) and controls without phenolic acid were incubated for 14 days at $25^{\circ} \mathrm{C}$. Fungal biomass of $F$. langsethiae and F. sporotrichioides was not reduced by the phenolic acids. However, biosynthesis of T-2 toxin by F. langsethiae was significantly reduced by chlorogenic $(23.1 \%)$ and ferulic $(26.5 \%)$ acids. Production of T-2 by F. sporotrichioides also decreased with ferulic acid by $23 \%(p<0.05)$. In contrast, $p$-coumaric acid significantly stimulated the production of T-2 and HT-2 toxins for both strains. A kinetic study of $F$. langsethiae with $1 \mathrm{mM}$ ferulic acid showed a significant decrease in fungal biomass, whereas T-2 production increased after 10 days of incubation. The study of gene expression in ferulic supplemented cultures of $F$. langsethiae revealed a significant inhibition for Tri5, Tri6 and Tri12 genes, while for Tri16 the decrease in gene expression was not statistically significant. Overall, results indicated that phenolic acids had a variable effect on fungal growth and mycotoxin production, depending on the strain and the concentration and type of phenolic acid assayed.
\end{abstract}

Keywords: Fusarium langsethiae; Fusarium sporotrichioides; type A trichothecene; phenolic acid; Tri gene

\section{Introduction}

T-2 toxin (T-2) and HT-2 toxin (HT-2) are two closely related type A trichothecenes produced by several Fusarium species, mainly F. langsethiae and F. sporotrichioides [1,2]. These mycotoxins may contaminate harvested grain and feed and food products derived thereof. Their presence in cereals such as oat, barley, maize and wheat in cold temperate climates of northern Europe is well documented [3-6]. T-2 and HT-2 are commonly found together in cereals, as HT-2 is a diacetylated form of T-2 [4,7]. T-2 toxin and HT-2 toxin are toxic to all animal species, as well as to humans. The toxic effects of T-2 and HT-2 include the inhibition of protein synthesis and hematopoiesis, lymphoid depletion and necrotic lesions. The consumption of moldy grains with high levels of T-2 has been associated with human intoxications, such as the Alimentary Toxic Aleukia (ATA), producing sepsis, hemorrhages and pancytopenia $[8,9]$. 
In view of their high toxicity and known presence in food and feed, T-2 and HT-2 toxins are considered a potential risk for animal and human health. Thus, a Tolerable Daily Intake (TDI) of $100 \mathrm{ng} / \mathrm{kg}$ body weight for the sum of T-2 and HT-2 has been established, which is the lowest TDI of all Fusarium toxins.

Conversely, even though maximum limits of T-2 and HT-2 in food products are not yet established due to the limited information available on their occurrence, the European Commission (EC) has recently published indicative values for the sum of T-2 and HT-2 in cereals and cereal products above which investigations should be performed [10]. The recommended levels specified for cereal grains and products for human consumption range from $15 \mu \mathrm{g} / \mathrm{kg}$ in cereal-based foods for infants and young children to $200 \mu \mathrm{g} / \mathrm{kg}$ in oat, oat bran and flaked oats.

Once they have been synthesized, mycotoxins are very difficult to remove, therefore control and prevention strategies in the field and during storage are important to minimize their presence in food and feed. To this end, fungicides have been widely used to control Fusarium growth and mycotoxin production both pre and post-harvest. However, legislation concerning the use of these compounds has become tougher in recent years. Besides, most of the synthetic fungicides are frequently perceived to present a hazard to human health and to the environment [11].

Therefore, there have been intensive efforts to find natural alternatives to fungicides that could be used safely to partially or completely inhibit the growth of fungi as well as mycotoxin biosynthesis. Among natural products, the ones which have been the most studied are the phenolic acids, which represent the most common form of phenolic compounds in whole grains and have been reported as in vitro inhibitors of Fusarium spp. growth [12-16] as well as other fungal genus such as Aspergillus spp. [17]. As regards in vitro studies on mycotoxin production, phenolic acids showed a significant inhibitory effect on type B trichothecenes [16,18-20] and fumonisin B1 [11,14,21].

Real-time PCR has been widely used to evaluate the effects of fungicide treatments on fungal gene expression. The most representative genes associated with type A trichothecene biosynthesis have been described in several studies [22,23]. Trichothecene production is driven by the transcription of the Tri5 gene which expression is positively regulated by Tri6 transcriptional regulator. Tri16 gene is responsible for structural variation at C-8 of type A trichothecenes, while Tri12 is a transporter gene.

The effect of fungicides [6,24] and of environmental conditions such as water activity and temperature $[3,25,26]$ on fungal growth and T-2 and HT-2 mycotoxin production by F. langsethiae was the subject of recent investigations. However, as far as we know, no studies have been performed yet on the effects of phenolic acids on the growth and production of T-2 and HT-2 toxins by F. langsethiae and F. sporotrichioides, as well as dealing with the effects of phenolic acids at the transcriptional level on the genes involved in type A trichothecene biosynthesis.

A large diversity in phenolic acids content has been found among cultivars of many cereal crops depending on genetic, environmental and agronomic variability. Therefore, breeding cereal varieties with increased content in bioactive phenolic acids could be a useful pre-harvest strategy for the control of toxigenic fungi [27]. However, it is important to determine the potential of phenolic acids on the inhibition of fungal growth and toxin production before extensive breeding work is initiated.

Therefore, the aims of the present study were to determine the in vitro effects of four phenolic acids (caffeic, chlorogenic, ferulic and p-coumaric), on the fungal biomass and T-2 and HT-2 toxin production by F. langsethiae and F. sporotrichioides. Additionally, a study of gene expression was carried out on the genes involved in type A trichothecene biosynthesis as affected by ferulic acid.

\section{Results}

\subsection{Effect of Phenolic Acids on Fungal Growth}

Dry fungal biomass of F. langsethiae INRA 466 and F. sporotrichioides INRA 101 cultivated in Mycotoxin Synthetic (MS) medium supplemented or not with $0.5 \mathrm{mM}$ phenolic acids is reported in Table 1. With the experimental conditions and Fusarium strains chosen for the present study, 
higher biomass values were obtained in the control for F. sporotrichioides (28.83 $\mathrm{mg}$ ) as compared to F. langsethiae (19.67 mg). The biomass of both Fusarium species was significantly affected by most of the tested phenolic acid treatments at $0.5 \mathrm{mM}$. Thus, caffeic, chlorogenic and ferulic acids increased fungal biomass of F. langsethiae $(p<0.05)$, while the biomass of F. sporotrichioides increased with caffeic, ferulic and $p$-coumaric acids $(p<0.05)$.

Table 1. Effect of phenolic acids at concentration of $0.5 \mathrm{mM}$ on the dry fungal biomass of cultures incubated at $25{ }^{\circ} \mathrm{C}$ for 14 days. Values expressed as mean values \pm standard deviation (SD) of three biological replications.

\begin{tabular}{ccc}
\hline \multirow{2}{*}{ Phenolic Acid } & \multicolumn{2}{c}{ Dry Fungal Biomass (mg) } \\
\cline { 2 - 3 } & F. langsethiae & F. sporotrichioides \\
\hline Control & $19.67 \pm 0.70$ & $28.83 \pm 0.26$ \\
Caffeic acid & $26.03 * \pm 1.79$ & $31.97 * \pm 0.48$ \\
Chlorogenic acid & $24.53 * \pm 0.55$ & $29.42 \pm 0.43$ \\
Ferulic acid & $24.27^{*} \pm 1.42$ & $33.74 * \pm 1.16$ \\
p-Coumaric acid & $19.63 \pm 1.82$ & $30.64 * \pm 0.87$ \\
\hline
\end{tabular}

* Significant difference with respect to control according to $t$-test $(p<0.05)$.

As regards to the kinetic study of F. langsethiae, fungal growth was significantly reduced $(p<0.05)$ with ferulic acid at $1 \mathrm{mM}$ after 6, 10 and 14 days of incubation (Table 2). The higher fungal biomass was achieved after 14 days of incubation, when the control fungal biomass reached $24.7 \mathrm{mg}$, while that with ferulic acid amounted to $21.1 \mathrm{mg}$. In addition, in 3-day old broths, only $1 \%$ of the added ferulic acid was quantified in liquid media. Ferulic acid was undetectable after 6 days of incubation.

Table 2. Effect of ferulic acid $(1 \mathrm{mM})$ on dry fungal biomass of $F$. langsethiae incubated at $25^{\circ} \mathrm{C}$. Values expressed as mean values \pm SD of three biological replications.

\begin{tabular}{ccc}
\hline \multirow{2}{*}{ Time (days) } & \multicolumn{2}{c}{ Dry Fungal Biomass (mg) } \\
\cline { 2 - 3 } & Control & Ferulic Acid \\
\hline 3 & $6.92 \pm 1.49$ & $5.07 \pm 1.68$ \\
6 & $17.10 \pm 1.21$ & $14.51^{*} \pm 0.71$ \\
10 & $22.29 \pm 0.75$ & $17.83^{*} \pm 1.31$ \\
14 & $24.70 \pm 0.49$ & $21.11^{*} \pm 0.65$ \\
\hline
\end{tabular}

* Significant difference with respect to control according to $t$-test $(p<0.05)$.

\subsection{Effect of Phenolic Acids on the Production of T-2 and HT-2 by Fusarium Strains}

T-2 production was higher than HT-2 in all controls and treatments with phenolic acids in both fungal species under study. Supplementation with $0.5 \mathrm{mM}$ chlorogenic and ferulic acids significantly reduced T-2 biosynthesis by F. langsethiae by $23 \%$ and $26 \%$, respectively (Figure 1), while HT-2 remained essentially unchanged. Conversely, $p$-coumaric acid increased T-2 and HT-2 production by approximately 2-fold. With regard to toxin production by F. sporotrichioides (Figure 2), the tested strain produced up to $2.47 \mathrm{mg} / \mathrm{g}$ of T-2 and $0.79 \mathrm{mg} / \mathrm{g}$ of HT-2, which is higher than the amount synthetized by the F. langsethiae strain. Ferulic acid reduced T-2 production by $23 \%(p<0.05)$, while $p$-coumaric acid significantly stimulated the production of T-2 and HT-2 by $24 \%$ and $47 \%$, respectively $(p<0.05)$. 


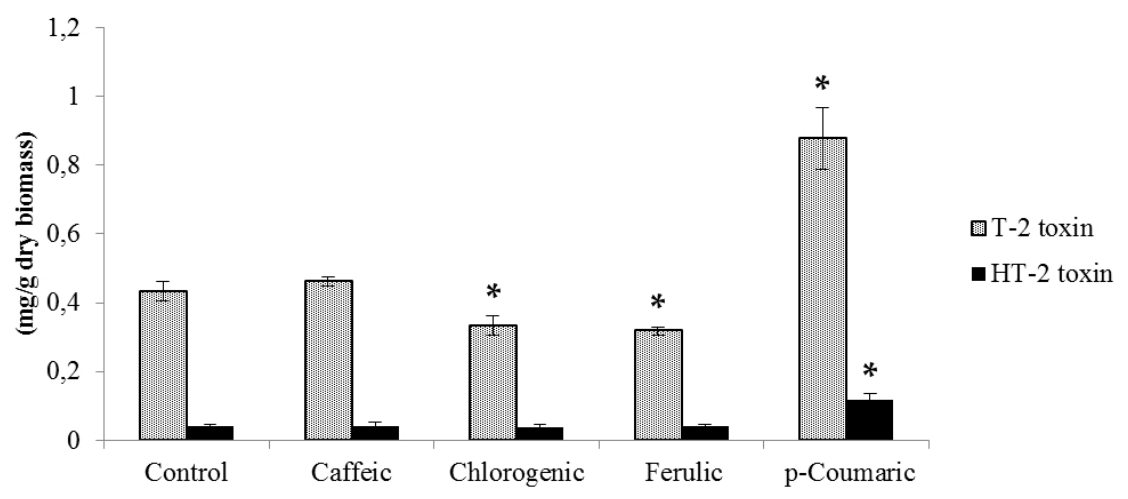

Figure 1. T-2 and HT-2 toxin production by F. langsethiae at $0.5 \mathrm{mM}$ of each phenolic acid incubated at $25^{\circ} \mathrm{C}$ for 14 days. Values expressed as mean values \pm SD of three biological replications. The sign * indicates significant difference with respect to control according to $t$-test $(p<0.05)$.

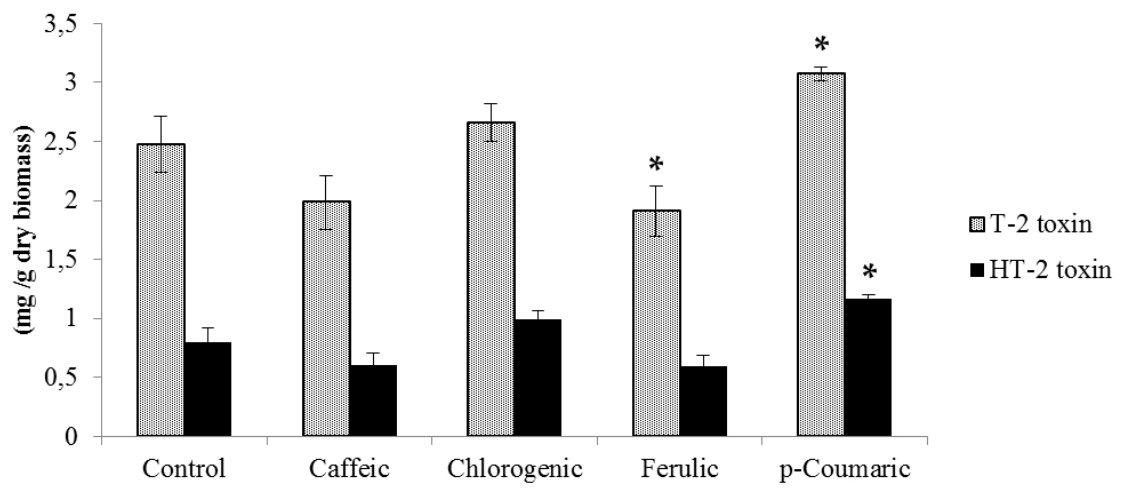

Figure 2. T-2 and HT-2 toxin production by F. sporotrichioides at $0.5 \mathrm{mM}$ of each phenolic acid incubated at $25{ }^{\circ} \mathrm{C}$ for 14 days. Values expressed as mean values \pm SD of three biological replications. The sign * indicates significant difference with respect to control according to $t$-test $(p<0.05)$.

In the kinetic study of $F$. langsethiae, T-2 was detected after 3 days of incubation whereas HT-2 biosynthesis started later, between 6 and 10 days of incubation in all plates (both control and treatment with ferulic acid) (Figure 3). After 3 and 6 days of incubation, plates treated with ferulic acid at $1 \mathrm{mM}$ produced significantly less T-2 than controls did $(p<0.05)$. However, after 10 and 14 days of incubation, treated plates were characterized by significantly higher levels of T-2 $(0.60 \mathrm{mg} / \mathrm{g}$ after 14 days) compared to controls $(0.37 \mathrm{mg} / \mathrm{g}$ after 14 days) $(p<0.05)$. The contrary effect was observed for HT-2 levels after 10 and 14 days of incubation, which were significantly lower $(p<0.05)$ in ferulic acid plates $(0.025 \mathrm{mg} / \mathrm{g}$ after 14 days) than in control plates $(0.039 \mathrm{mg} / \mathrm{g}$ after 14 days $)$. As in the previous in vitro study performed with $0.5 \mathrm{mM}$ of phenolic acid, concentration of T-2 was higher than that of HT-2 in all plates.
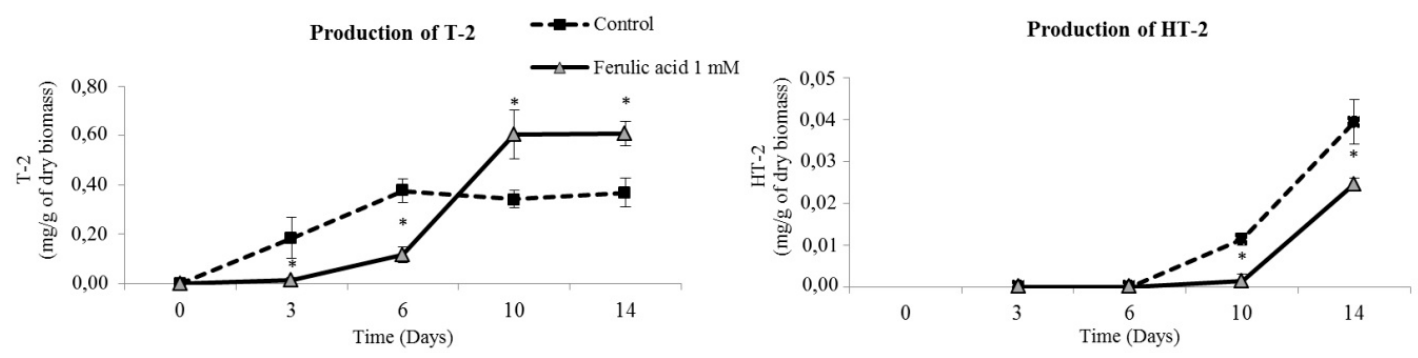

Figure 3. Kinetics of T-2 and HT-2 toxin by F. langsethiae INRA 466 at $1 \mathrm{mM}$ of ferulic acid incubated at $25^{\circ} \mathrm{C}$. The sign * indicates significant difference with respect to control according to $t$-test $(p<0.05)$. 


\subsection{Effect of Ferulic Acid on Tri Gene Expression by F. langsethiae}

To evaluate effects at the transcriptional level involved in type A trichothecene biosynthesis inhibition by ferulic acid, the expression of several representative genes involved in the biosynthesis pathway (Tri5, Tri6, Tri12 and Tri16) was analyzed. Expression of all the studied Tri genes was lower in ferulic acid supplemented culture as compared with control. A significant inhibition of expression induced by ferulic acid was observed for Tri5, Tri6 and Tri12 genes, while for Tri16 gene the decrease was not statistically significant.

The regulation factors obtained for the target gene in the ferulic acid supplemented culture relative to the control culture were calculated using the REST software. Thus, the biosynthesis gene Tri5 was expressed 2.85 times less in ferulic acid treated cultures, while expression of the transcription factor Tri6 was reduced 6.5 times. Additionally, the expression of the Tri12 (transporter) and the Tri16 (C-8 modification) genes were reduced 2.4 and 1.8 times, respectively, in ferulic acid treated cultures. This expression pattern suggests that, in ferulic acid supplemented cultures, the decrease in toxin production could result from a decrease in the level of Tri gene expression (see Figure 4).

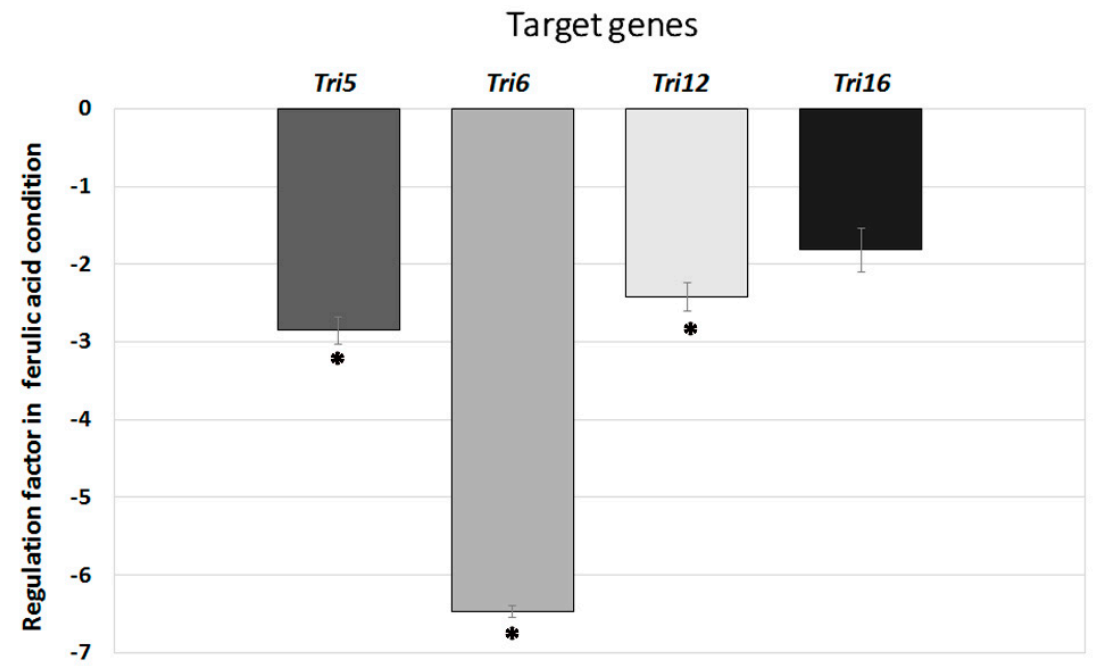

Figure 4. Regulation factors obtained for Tri genes in culture supplemented with ferulic acid relative to the control culture. The sign * indicates a significant regulation according to $t$-test $(p<0.05)$.

\section{Discussion}

The present study has evaluated the efficacy of four phenolic acids (caffeic, chlorogenic, ferulic and $p$-coumaric) against F. sporotrichioides and F. langsethiae in relation to both growth and T-2 and HT-2 production.

Most phenolic acids tested at $0.5 \mathrm{mM}$ increased the fungal biomass of $F$. langsethiae and F. sporotrichioides. However, the effects of phenolic acids on T-2 and HT-2 production were dependent on the type of phenolic acid and the strain. Thus, ferulic acid showed a clear inhibitory effect on T-2 production by F. langsethiae and F. sporotrichioides, chlorogenic acid only inhibited T-2 synthesis by F. langsethiae, while caffeic acid showed little effects on toxin production. Finally, $p$-coumaric acid stimulated T-2 and HT-2 production by both F. sporotrichioides and F. langsethiae.

As far as we know, there are no available studies on the effects of phenolic acids on the growth of F. sporotrichioides and F. langsethiae and their impact on T-2 and HT-2 production. However, recent in vitro studies have been performed on other Fusarium species and mycotoxins. Thus, as observed in the present work, other studies testing the effects of ferulic and $p$-coumaric acids on type B trichothecene production also obtained variable results depending on the strain, type and concentration of phenolic acid. Boutigny et al. [19] reported an inhibitory effect of ferulic acid on fungal growth and fully inhibition of type B trichothecene production by F. culmorum in MS medium at $2.5 \mathrm{mM}$. However, 
lower concentrations (from 0.1 to $1 \mathrm{mM}$ ) did not affect fungal growth, while mycotoxin production was highly inhibited, ranging from $68 \%$ to $99 \%$ inhibition. This result is in accordance with the present study, as $0.5 \mathrm{mM}$ of ferulic acid reduced T-2 synthesis but increased the fungal growth. On the contrary, Picot et al. [21] found that the same concentration of ferulic acid did not affect fungal growth or fumonisin production by F. verticillioides in Glucose Yeast Extract Peptone (GYEP) medium; though higher concentrations $(1 \mathrm{mM})$ did have an inhibitory effect ( $53 \%-86 \%)$ on fumonisin biosynthesis.

What's more, it is worth mentioning that the stimulating effect on mycotoxin production observed with $p$-coumaric acid had been previously described by Ponts et al. [16], who reported that GYEP medium supplemented with $p$-coumaric acid at $0.5 \mathrm{mM}$ enhanced type $\mathrm{B}$ trichothecene biosynthesis by F. graminearum.

In the kinetic study of $F$. langsethiae, ferulic acid at $1 \mathrm{mM}$ reduced fungal growth at all times of incubation. However, while T-2 and HT-2 production were significantly reduced at 3 and 6 days of incubation, this decreasing trend changed after 10 days of culture. At this time of incubation, toxin production was stimulated and concentrations of T-2 and HT-2 in the treated medium were significantly higher $(p<0.05)$ than controls. This shift in the trend could be related to the levels of ferulic acid in the treated plates, which were decreasing from the beginning of the incubation period. Thus, in the third day only $1 \%$ of added ferulic acid was still remaining while went undetected on the sixth day. This finding could be hypothetically explained because ferulic acid is being depleted while interfering with fungal metabolism [19]. Once ferulic acid has been fully metabolized, the fungus has no obstacles to produce T-2 and HT-2. Similarly to what was observed with F. graminearum and DON production [16], $1 \mathrm{mM}$ ferulic acid can significantly inhibit the breakdown of hydrogen peroxide by the Fusarium strain and therefore the enhancement of T2/HT2 accumulation is consistent with the activating effect frequently assigned to hydrogen peroxide on mycotoxin production [28].

Comparing these results with those obtained in the in vitro study with ferulic acid at $0.5 \mathrm{mM}$, it was observed that lower doses stimulated fungal growth and inhibited toxin production, whereas higher doses had the contrary effect. These findings might suggest that sublethal doses of ferulic acid could have an inhibitory effect of toxin production and no effects on fungal growth.

At this concern, Ferrochio et al. [11] reported that $1 \mathrm{mM}$ of ferulic acid in maize based medium, stimulated both fungal growth and fumonisin production. However, higher concentrations $(20 \mathrm{mM}$ and $25 \mathrm{mM}$ ) significantly reduced both $F$. verticillioides growth and fumonisin production. Lower concentrations of $10 \mathrm{mM}$ stimulated fumonisin production while still inhibiting fungal growth.

In relation to the strains assayed, in the present study, F. langsethiae was more sensitive to phenolic acids (chlorogenic and ferulic acid reduced toxin production) than F. sporotrichioides (only affected with ferulic acid). In addition, F. sporotrichioides strain produced higher levels of T-2 and HT-2 than F. langsethiae, regardless of the treatment with phenolic acid. This is in accordance with a recent in vitro study by Kokonnen et al. [1] in which F. sporotrichioides strains produced higher concentrations of T-2 and HT-2 than F. langsethiae did.

Furthermore, the transcription of the most representative Tri genes associated with the type A trichothecene biosynthetic pathway (Tri5, Tri6, Tri12 and Tri16) was investigated. Results showed a decrease in the relative quantity of Tri genes transcription in ferulic supplemented cultures of F. langsethiae. This suggests for the first time that inhibition of toxin synthesis by this phenolic acid could be regulated at the transcriptional level. Comparable results have been obtained by Boutigny et al. [20] suggesting that natural phenolic acids from wheat bran inhibit Fusarium culmorum type B trichothecene biosynthesis in vitro by repressing Tri gene expression.

Accordingly to the results obtained in this work and in addition to those described by Ferrochio et al. [11], it seems that mechanisms of inhibition of Fusarium growth by phenolic acids are independent of mechanisms of inhibition behind mycotoxin production. At this concern, our data support the results of Boutigny et al. [19] who suggested that type B trichothecenes biosynthesis could be regulated at a transcriptional level, therefore having a regulatory mechanism different from that of fungal growth. Nevertheless, other authors suggested a relationship between growth and mycotoxin 
production as affected by phenolic compounds $[29,30]$. All in all, more research is required to elucidate the effects of phenolic acids and the way they regulate fungal growth and mycotoxin synthesis.

\section{Materials and Methods}

\subsection{Materials}

Caffeic, chlorogenic, ferulic and p-coumaric acids were obtained from commercial pure powders purchased from Sigma-Aldrich (Saint-Quentin Fallavier, France). These phenolic acids were selected because of their natural abundance in cereal grains. Standard solutions of T-2 $(10 \mu \mathrm{g} / \mathrm{mL})$ and HT-2 $(10 \mu \mathrm{g} / \mathrm{mL})$ toxins in acetonitrile were obtained from Biopure Standard Solutions (Romer Labs, Tulln, Austria) and stocked at $-20^{\circ} \mathrm{C}$ until use.

\subsection{Spore Suspension of Fusarium Strains}

F. langsethiae INRA 466 and F. sporotrichioides INRA 101 were obtained from the INRA-Bordeaux MycSA collection deposited in the International Center for Microbial Resources - Filamentous Fungi (CIRM-CF, http://www6. inra.fr/cirm_eng/Filamentous-Fungi/Strains-catalogue, Marseille, France). Stock cultures were maintained at $4{ }^{\circ} \mathrm{C}$ on Potato Dextrose Agar (PDA) (BD Difco ${ }^{\mathrm{TM}}$, Illkirch, France) Petri plates. The strains were inoculated and incubated on PDA slants tubes at $25^{\circ} \mathrm{C}$ for seven days. The spore suspension was prepared for each strain by adding $6 \mathrm{~mL}$ of sterile distilled water to grown agar slants with gentle shaking. The spore suspension was collected directly in another tube and spores were counted on a Thoma cell.

\subsection{Antifungal Activity Assay}

Liquid culture assays were performed in a Mycotoxin Synthetic medium (MS medium) $\left(\mathrm{KH}_{2} \mathrm{PO}_{4}\right.$, $0.5 \mathrm{~g} / \mathrm{L} ; \mathrm{K}_{2} \mathrm{HPO}_{4}, 0.6 \mathrm{~g} / \mathrm{L} ; \mathrm{MgSO}_{4}, 0.017 \mathrm{~g} / \mathrm{L} ;\left(\mathrm{NH}_{4}\right)_{2} \mathrm{SO}_{4}, 1 \mathrm{~g} / \mathrm{L} ;$ glucose, $20 \mathrm{~g} / \mathrm{L}$; biotin, $0.1 \mathrm{mg} / \mathrm{L}$ and $0.1 \mathrm{~mL} / \mathrm{L}$ of Vogel mineral salts solution [31]) as described by Boutigny et al. [20]. Phenolic acids were dissolved directly in MS medium to achieve a final concentration of $0.5 \mathrm{mM}$. An $8 \mathrm{~mL}$ aliquot of supplemented MS medium was placed in sterile Petri dishes ( $55 \mathrm{~mm}$ diameter). A control group with no phenolic acid was also prepared. Confirmation of phenolic acid proper supplementation was performed by HPLC- DAD analysis.

Supplemented Petri plates were inoculated with $100 \mu \mathrm{L}$ spore suspension $\left(10^{6}\right.$ spores $\left./ \mathrm{mL}\right)$ of F. langsethiae and F. sporotrichioides and incubated at $25^{\circ} \mathrm{C}$ in the dark for 14 days. Cultures were prepared in triplicate. After incubation, cultures were collected in tubes and centrifuged at $3000 \mathrm{~g}$ for $10 \mathrm{~min}$ in order to separate culture medium from mycelium. Supernatant was stored at $-20^{\circ} \mathrm{C}$ before quantification of mycotoxins. The mycelium was stored at $-80^{\circ} \mathrm{C}$. Fusarium spp. biomass was measured by weighing the mycelia after $48 \mathrm{~h}$ of lyophilization. Fungal growth was determined as dry weight of mycelium. The $\mathrm{pH}$ of culture media was measured before and after incubation in order to check that $\mathrm{pH}$ did not change compared to the control.

Following the previous in vitro study described above, a kinetic study of T-2 and HT-2 toxin production and fungal growth by F. langsethiae INRA 466 was performed to determine the effects of supplementation with $1 \mathrm{mM}$ ferulic acid. Ferulic acid was selected because it is the most abundant phenolic acid in cereal grains. Petri plates ( $55 \mathrm{~mm}$ diameter) containing MS medium supplemented with $1 \mathrm{mM}$ of ferulic acid were inoculated with a spore suspension of $F$. langsethiae $\left(10^{6}\right.$ spores $\left./ \mathrm{mL}\right)$ and incubated at $25{ }^{\circ} \mathrm{C}$ with four different incubation times $(3,6,10$ and 14 days). Cultures were prepared in triplicate. Fungal growth, T-2 and HT-2 toxin production and ferulic acid concentration were determined at these different incubation times.

\subsection{Extraction of Total RNA, Preparation of $c D N A$ and RT-PCR Analysis}

Total RNA was extracted from 3-day-old mycelium from F. langsethiae 466 grown in medium with or without $0.5 \mathrm{mM}$ ferulic acid. Four biological replications were prepared for each condition. Fifty 
milligrams of frozen mycelium were grinded using a TissueLyser II ${ }^{\circledR}$ (QIAGEN, Courtaboeuf, France) for $1 \mathrm{~min}$ at $30 \mathrm{~Hz}$ before RNA extraction using the RNeasy Plant Mini Kit (QIAGEN), according to the manufacturer's instructions. Removal of residual DNA using treatment with the DNA-free ${ }^{\mathrm{TM}}$ Kit (Ambion by Life Technology SAS, Carlsbad, USA) was performed according to recommendations of the manufacturer. One microgram of total RNA was reverse transcribed using the SuperScript III First-Strand Synthesis System for RT-PCR (Invitrogen, Life Technology SAS), with oligo(dT)20 primers, according to the manufacturer's instructions. The cDNA obtained were diluted five folds and stored at $-20^{\circ} \mathrm{C}$. Gene expression analysis was performed using $1 \mu \mathrm{L}$ of each cDNA preparation (corresponding to $10 \mathrm{ng}$ of total RNA input in the reverse transcription) in a $10 \mu \mathrm{L}$ reaction volume, using the QuantiFast SYBR ${ }^{\circledR}$ Green PCR kit (QIAGEN). A LightCycler ${ }^{\circledR} 2.0$ system equipped with the LightCycler software 4.0.5 (Roche Diagnostics, Rotkreuz, Switzerland) was used to carry out the reactions. Amplification efficiency (Table 3) was evaluated for each gene with serial dilutions of the pooled cDNA samples and data analysis was performed as previously described by Ponts et al. [16]. Expression levels of the studied genes (Tri5, Tri6, Tri12 and Tri16) were normalized to the expression of the reference genes $\beta$-tubulin and citrate synthase (Table 3). Statistical analysis was performed using the REST R-software (Relative Expression Software Tool).

Table 3. Studied genes, primers used for qPCR analysis and PCR efficiency.

\begin{tabular}{|c|c|c|c|c|}
\hline Gene & $\begin{array}{l}\text { Accession No. } \\
\text { Genbank }\end{array}$ & $\begin{array}{l}\text { Forward Primer } \\
\text { Sequence } 5^{\prime}-3^{\prime}\end{array}$ & $\begin{array}{l}\text { Reverse Primer } \\
\text { Sequence } 5^{\prime}-3^{\prime}\end{array}$ & PCR Efficiency \\
\hline B-tubulin & AF212817 & $\begin{array}{c}\text { GGTAACCAAATCGG } \\
\text { TGCTGCTTTC }\end{array}$ & $\begin{array}{l}\text { GATTGACCGAAAAC } \\
\text { G AAGTTG }\end{array}$ & 1.97 \\
\hline Citrate synthase & XM_011318922 & $\begin{array}{c}\text { GGCTCACCGAGTTCA } \\
\text { AGAAG }\end{array}$ & $\begin{array}{c}\text { CTTCTCTTGGGCAA } \\
\text { AAGTGC }\end{array}$ & 1.97 \\
\hline Tri5 & AF449792.1 & $\begin{array}{c}\text { CTATTCCTTGAGATT } \\
\text { ACAT }\end{array}$ & $\begin{array}{l}\text { CCTTGTAGAATGAC } \\
\text { ATAAGA }\end{array}$ & 2.00 \\
\hline Tri6 & $\begin{array}{c}\text { JXCE01000103.1; } \\
\text { locus tag } \\
\text { FLAG_12235 }\end{array}$ & $\begin{array}{c}\text { CGCTTTCGAATATGG } \\
\text { TGGTT }\end{array}$ & $\begin{array}{l}\text { CCTACGGTGGAGCC } \\
\text { TACAAA }\end{array}$ & 2.06 \\
\hline Tri12 & $\begin{array}{c}\text { JXCE01000103.1; } \\
\text { locus tag } \\
\text { FLAG_05858 }\end{array}$ & $\begin{array}{l}\text { GGGCTTGCATATCTT } \\
\text { GTGGT }\end{array}$ & $\begin{array}{l}\text { TTCGGCCCTATTCGT } \\
\text { ACAAC }\end{array}$ & 1.88 \\
\hline Tri16 & HQ594543.1 & $\begin{array}{c}\text { GGTCTGGTCTAATCT } \\
\text { TACA }\end{array}$ & $\begin{array}{l}\text { CACGACATTACCCA } \\
\text { TATAAG }\end{array}$ & 2.02 \\
\hline
\end{tabular}

\subsection{Analysis of T-2 and HT-2 Toxins by HPLC-MS/MS}

A representative sample of culture medium was diluted with $50 \%$ methanol, shaken with a Vortex mixer and filtered through $0.45 \mu \mathrm{m}, 15 \mathrm{~mm}$ diameter RC Phenex ${ }^{\mathrm{TM}}$ filter syringe (Phenomenex, Le Pecq, France) before HPLC-MS/MS analysis.

Concentrations of T-2 and HT-2 toxins in liquid cultures were determined using a QTrap 2000 LC/MS/MS system (AB Sciex, Les Ulis, France) equipped with a Turbo Ion Spray ESI source and a 1100 Series HPLC system (Agilent Technology, Massy, France). A Zorbax Eclipse XDB C18 column $(150 \mathrm{~mm} \times 2.1 \mathrm{~m}, 0.5 \mu \mathrm{m})$ (Agilent, France) was used; the temperature of the column was maintained at $30{ }^{\circ} \mathrm{C}$. A gradient elution, consisting in $10 \%(v / v)$ methanol in high purity water with $5 \mathrm{mM}$ ammonium acetate (solvent $A$ ) and $90 \%(v / v)$ methanol in high purity water with $5 \mathrm{mM}$ ammonium acetate (solvent B) was employed as follows: $10 \%-70 \%$ B in $10 \mathrm{~min}, 70 \%-100 \%$ B in $2 \mathrm{~min}$, isocratic elution $100 \%$ B for $8 \mathrm{~min}, 100 \%-10 \%$ B for $1 \mathrm{~min}$, and $9 \mathrm{~min}$ post-run reconditioning. The flow rate was kept at $300 \mu \mathrm{L} / \mathrm{min}$ and the injection volume was $20 \mu \mathrm{L}$.

Quantification was performed on MRM positive mode by monitoring one transition for each analyte: $m / z 442.2 / 215.1$ for HT-2 and $m / z 484.2 / 215.3$ for T-2 toxin. The electrospray interface was used 
at $400{ }^{\circ} \mathrm{C}$ with the following settings: auxiliary gas, 60 p.s.i.; ion spray voltage, $4500 \mathrm{~V}$; declustering potential, 36 and $46 \mathrm{~V}$ for HT-2 and T-2, entrance potential, 8.5 and $5.5 \mathrm{~V}$ for HT-2 and T-2; collision energy, 19 and $25 \mathrm{eV}$ for HT-2 and T-2 respectively.

External calibration was performed to quantify T-2 and HT-2 toxins $(25,50,100,250,500$ and $1000 \mu \mathrm{g} / \mathrm{L}$ ). Results were converted in $\mu \mathrm{g} / \mathrm{g}$ of dry fungal biomass. Data are reported as mean values \pm standard deviation (SD) of three biological replicates.

\subsection{Analysis of Phenolic Acids by HPLC-DAD}

Analysis of phenolic acids was performed to confirm the proper supplementation of MS media as well as to determine the evolution of ferulic acid concentration in the kinetic study. Extraction and determination of phenolic acids was performed according to Boutigny et al. [20] with some modifications: culture media were diluted with methanol:water $(1: 1, v / v)$ and filtered through a $0.45 \mu \mathrm{m}$ syringe filter (Phenomenex) before HPLC-DAD analysis. Separation of phenolic acids was achieved on a Kinetex XB-C18 column $(150 \mathrm{~mm} \times 4.6 \mathrm{~mm}, 2.6 \mu \mathrm{m})$ (Phenomenex) maintained at $35^{\circ} \mathrm{C}$. The mobile phase consisted of $2 \%$ formic acid in water $(v / v)$ (solvent $\mathrm{A}$ ) and acetonitrile (solvent B). Phenolic acids were separated by a gradient elution as follows: linear gradient from $5 \%$ to $11 \%$ B, 0-18 min; isocratic elution $11 \% \mathrm{~B}, 18-22 \mathrm{~min}$, linear gradient $11 \%$ to $40 \% \mathrm{~B}, 22-35 \mathrm{~min}$; linear gradient $40 \%$ to $90 \%$ B, 35-36 min; isocratic elution $90 \%$ B, 36-41 min; linear gradient from $90 \%$ to $5 \%$ $\mathrm{B}, 41-43 \mathrm{~min}$ and $5 \% \mathrm{~B}$ for $10 \mathrm{~min}$ post-run reconditioning. The injection volume was $5 \mu \mathrm{L}$. The flow rate was kept at $1 \mathrm{~mL} / \mathrm{min}$ for a total run time of $43 \mathrm{~min}$. The UV-VIS spectra were recorded from 190 to $500 \mathrm{~nm}$ and peak areas were measured at $320 \mathrm{~nm}$ for all phenolic acids. Quantification of phenolic acids was performed by using external calibration. Standard solutions of phenolic acids were prepared by dissolving adequate amounts of standards in methanol-water $(1: 1, v / v)$.

\subsection{Statistical Analysis}

Fungal growth and T-2 and HT-2 toxin production of Fusarium strains were analyzed statistically with SPSS software v. 20 (IBM Inc., Armonk, NY, USA), performing a t-Student test $(p<0.05)$ (control against treatment with phenolic acids).

\section{Conclusions}

The results obtained in this study suggest that phenolic acids at a dose of $0.5 \mathrm{mM}$ enhanced the fungal biomass of $F$. langsethiae and F. sporotrichioides, while the effects on T-2 and HT-2 production were dependent on the type of phenolic acid and the strain. Ferulic acid reduced T-2 production on both fungal species, while the synthesis of T-2 and HT-2 increased with p-coumaric. In the kinetic study, a higher dose of ferulic acid $(1 \mathrm{mM})$ reduced significantly fungal growth of $F$. langsethiae, but mycotoxin synthesis after 14 days of incubation was increased (T-2) or reduced (HT-2). In the study of the transcriptional activity a reduced Tri gene expression in ferulic supplemented cultures of F. langsethiae was observed. All these observations indicate that mechanisms for fungal growth and mycotoxin production may be independent. This study may help in further understanding the effects of phenolic acids on growth and toxin production by Fusarium fungi, as well as the effect on the trichothecene genes and their transcription.

Acknowledgments: Author Elena Ferruz acknowledges F. Richard-Forget for her acceptance for a 3-month research stay at INRA, UR 1264 MycSA (France). This research was also supported by the projects AGL2011-26808, AGL2014-57069-R, AGL2014-52648-REDT by MINECO/FEDER, DGA A01 and the European Social Fund. Author Elena Ferruz acknowledges a grant from Fundación Cuenca Villoro.

Author Contributions: F.R.-F., C.B. and V.A.-P. conceived and designed the experiments; E.F., V.A.-P., M.-N.B.-V. and G.M. performed the experiments on growth and toxin production; L.P.-G. and C.D. conducted the studies of gene expression; all authors analyzed the data; S.L. and A.A. wrote the paper. 
Conflicts of Interest: The authors declare no conflict of interest. The founding sponsors had no role in the design of the study; in the collection, analyses, or interpretation of data; in the writing of the manuscript, and in the decision to publish the results.

\section{References}

1. Kokkonen, M.; Medina, A.; Magan, N. Comparative study of water and temperature relations of growth and T-2/HT-2 toxin production by strains of Fusarium sporotrichioides and Fusarium langsethiae. World Mycotoxin J. 2012, 5, 365-372. [CrossRef]

2. Nazari, L.; Pattori, E.; Terzi, V.; Morcia, C.; Rossi, V. Influence of temperature on infection, growth, and mycotoxin production by Fusarium langsethiae and F. sporotrichioides in durum wheat. Food Microbiol. 2014, 39, 19-26. [CrossRef] [PubMed]

3. Medina, A.; Magan, N. Comparisons of water activity and temperature impacts on growth of Fusarium langsethiae strains from northern Europe on oat-based media. Int. J. Food Microbiol. 2010, 142, 365-369. [CrossRef] [PubMed]

4. Edwards, S.G.; Imathiu, S.M.; Ray, R.V.; Back, M.; Hare, M.C. Molecular studies to identify the Fusarium species responsible for HT-2 and T-2 mycotoxins in UK oats. Int. J. Food Microbiol. 2012, 156, 168-175. [CrossRef] [PubMed]

5. Lindblad, M.; Gidlund, A.; Sulyok, M.; Börjesson, T.; Krska, R.; Olsen, M.; Fredlund, E. Deoxynivalenol and other selected Fusarium toxins in Swedish wheat - occurrence and correlation to specific Fusarium species. Int. J. Food Microbiol. 2013, 167, 284-291. [CrossRef] [PubMed]

6. Mateo, E.V.; Valle-Algarra, F.M.; Mateo, R.; Jiménez, M.; Magan, N. Effect of fenpropimorph, prochloraz and tebuconazole on growth and production of T-2 and HT-2 toxins by Fusarium langsethiae in oat-based medium. Int. J. Food Microbiol. 2011, 151, 289-298. [CrossRef] [PubMed]

7. De Angelis, E.; Monaci, L.; Mackie, A.; Salt, L.; Visconti, A. Bioaccessibility of T-2 and HT-2 toxins in mycotoxin contaminated bread models submitted to in vitro human digestion. Innov. Food Sci. Emerg. 2014, 22, 248-256. [CrossRef]

8. EFSA (European Food Safety Authority). Scientific opinion on the risks for animal and public health related to the presence of T-2 and HT-2 toxin in food and feed. EFSA panel on contaminants in the food chain. EFSA J. 2011, 9, 2481.

9. Peraica, M.; Rašić, D. Review. The impact of mycotoxicoses on human history. Arh. Hig. Rada Toksiko. 2012, 63, 513-518. [CrossRef] [PubMed]

10. Commission Recommendation 2013/165/EU (OJ L91, p12, 03/04/2013) of 27 March 2013 on the Presence of T-2 and HT-2 Toxins in Cereals and Cereal Products.

11. Ferrochio, L.; Cendoya, E.; Farnochi, M.C.; Massad, W.; Ramírez, M.L. Evaluation of ability of ferulic acid to control growth and fumonisin production of Fusarium verticillioides and Fusarium proliferatum on maize based media. Int. J. Food Microbiol. 2013, 167, 215-220. [CrossRef] [PubMed]

12. McKeehen, J.D.; Busch, R.H.; Fulcher, R.G. Evaluation of wheat (Triticum aestivum L.) phenolic acids during grain development and their contribution to Fusarium resistance. J. Agric. Food Chem. 1999, 47, 1476-1482. [CrossRef] [PubMed]

13. Bily, A.C.; Reid, L.M.; Taylor, J.H.; Johnston, D.; Malouin, C.; Burt, A.J.; Bakan, B.; Regnault-Roger, C.; Pauls, K.P.; Arnason, J.T.; et al. Dehydrodimers of ferulic acid in maize grain pericarp and aleurone: resistance factors to Fusarium graminearum. Phytopathology 2003, 93, 712-719. [CrossRef] [PubMed]

14. Samapundo, S.; De Meulenaer, B.; Osei-Nimoh, D.; Lamboni, Y.; Debevere, J.; Devlieghere, F. Can phenolic compounds be used for the protection of corn fungal invasion and mycotoxin contamination during storage? Food Microbiol. 2007, 24, 465-473. [CrossRef] [PubMed]

15. Coma, V.; Portes, E.; Gardrat, C.; Richard-Forget, F.; Castellan, A. In vitro inhibitory effect of tetrahydrocurcuminoids on Fusarium proliferatum growth and fumonisin B1 biosynthesis. Food Addit. Contam. 2011, 2, 218-225. [CrossRef] [PubMed]

16. Ponts, N.; Pinsons-Gadais, L.; Boutigny, A.L.; Barreau, C.; Richard-Forget, F. Cinnamic-derived acids significantly affect Fusarium graminearum growth and in vitro synthesis of type B trichothecenes. Phytopathology 2011, 101, 929-934. [CrossRef] [PubMed] 
17. Nesci, A.; Etcheverry, M. Effect of natural maize phytochemicals on Aspergillus section Flavi sclerotia characteristics under different conditions of growth media and water potential. Fungal Ecol. 2009, 2, 44-51. [CrossRef]

18. Bakan, B.; Bily, A.C.; Melcion, D.; Cahagnier, B.; Regnault-Roger, C.; Philogène, B.J.; Richard-Molard, D. Possible role of plant phenolics in the production of trichothecenes by Fusarium graminearum strains on different fractions of maize kernels. J. Agric. Food Chem. 2003, 51, 2826-2831. [CrossRef] [PubMed]

19. Boutigny, A.L.; Barreau, C.; Atanasova-Pénichon, V.; Verdal-Bonnin, M.N.; Pinson-Gadais, L.; Richard-Forget, F. Ferulic acid, and efficient inhibitor of type B trichothecene biosynthesis and Tri gene expression in Fusarium liquid cultures. Mycol. Res. 2009, 3, 746-753. [CrossRef] [PubMed]

20. Boutigny, A.L.; Atanasova-Pénichon, V.; Benet, M.; Barreau, C.; Richard-Forget, F. Natural phenolic acids from wheat bran inhibit Fusarium culmorum trichothecene biosynthesis in vitro by repressing Tri gene expression. Eur. J. Plant Pathol. 2010, 127, 275-286. [CrossRef]

21. Picot, A.; Atanasova-Pénichon, V.; Pons, S.; Marchegay, G.; Barreau, C.; Pinson-Gadais, L.; Roucolle, J.; Daveau, F.; Caron, D.; Richard-Forget, F. Maize kernel antioxidants and their potential involvement in Fusarium ear rot resistance. J. Agric. Food Chem. 2013, 61, 3389-3395. [CrossRef] [PubMed]

22. Kimura, M.; Tokai, T.; Takahashi-Ando, N.; Ohsato, S.; Fujimura, M. Molecular and genetic studies of Fusarium trichothecene biosynthesis: Pathways, genes, and evolution. Biosci. Biotechnol. Biochem. 2007, 71, 2105-2123. [CrossRef] [PubMed]

23. McCormick, S.P.; Stanley, A.M.; Stover, N.A.; Alexander, N.J. Trichothecenes: From simple to complex mycotoxins. Toxins 2011, 3, 802-814. [CrossRef] [PubMed]

24. Mateo, E.M.; Valle-Algarra, F.M.; Jiménez, M.; Magan, N. Impact of three sterol-biosynthesis inhibitors on growth of Fusarium langsethiae and on T-2 and HT-2 toxin production in oat grain under different ecological conditions. Food Control 2013, 34, 521-529. [CrossRef]

25. Medina, A.; Magan, N. Temperature and water activity effects on production of T-2 and HT-2 by Fusarium langsethiae strains from north European countries. Food Microbiol. 2011, 28, 392-398. [CrossRef] [PubMed]

26. Mylona, K.; Magan, N. Fusarium langsethiae: Storage environment influences dry matter losses and T-2 and HT-2 toxin contamination of oats. J. Stored Prod. Res. 2011, 47, 321-327. [CrossRef]

27. Kaushik, P.; Andújar, I.; Vilanova, S.; Plazas, M.; Gramazio, P.; Herraiz, F.J.; Brar, N.S.; Prohens, J. Breeding Vegetables with Increased Content in Bioactive Phenolic Acids. Molecules 2015, 20, 18464-18481. [CrossRef] [PubMed]

28. Montibus, M.; Pinson-Gadais, L.; Richard-Forget, F.; Barreau, C.; Ponts, N. Coupling of transcriptional response to oxidative stress and secondary metabolism regulation in filamentous fungi. Crit. Rev. Microbiol. 2015, 41, 295-308. [CrossRef] [PubMed]

29. Torres, A.; Ramírez, M.L.; Arroyo, M.; Chulze, S.; Magan, N. Potential for control of growth and fumonisin production by Fusarium verticillioides and F. proliferatum on irradiated maize grain using anti-oxidants. Int. J. Food Microbiol. 2003, 83, 319-324. [CrossRef]

30. Dambolena, J.S.; Zygadlo, J.A.; Rubinstein, H.R. Antifumonisin activity of natural phenolic compounds. A structure- property-activity relationship study. Int. J. Food Microbiol. 2011, 145, 140-146. [CrossRef] [PubMed]

31. Vogel, H.J. A convenient growth medium for Neurospora (Medium N). Microb. Gen. Bull. 1956, 13, 42-43.

Sample Availability: Samples of the compounds are not available from the authors.

(C) 2016 by the authors; licensee MDPI, Basel, Switzerland. This article is an open access article distributed under the terms and conditions of the Creative Commons by Attribution (CC-BY) license (http://creativecommons.org/licenses/by/4.0/). 